\title{
A Numerical Study of a Class of LES Models
}

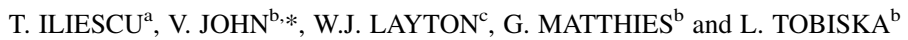 \\ ${ }^{a}$ Department of Mathematics, Virginia Polytechnic Institute and State University, Blacksburg, VA 24061, USA; \\ ${ }^{\mathrm{b}}$ Institut Für Analysis und Numerik, Otto-von-Guericke-Universität Magdeburg, Postfach 4120, 39016 Magdeburg, Germany; \\ ${ }^{\mathrm{c}}$ Department of Mathematics, University of Pittsburgh, Pittsburgh, PA 15260, USA
}

(Received 17 May 2001)

\begin{abstract}
This paper tests if two related types of LES models satisfy some simple necessary conditions for acceptability: replication of laminar flows and boundedness of total kinetic energy. The considered LES models are based on the approximation of the Fourier transform of the Gaussian filter by a simpler function. One uses a Taylor polynomial approximation (Taylor LES model), whereas the other model is obtained by a rational approximation (rational LES model). The numerical experiments at high Reynolds number 2D and 3D driven cavity flows show a blow up of the total kinetic energy of the solutions computed with the Taylor LES model. The details of the calculations and the review of this model's derivation point to this blow up being clearly a shortcoming of the model. In contrast, the rational LES model gives solutions with bounded total kinetic energy. In addition, the large eddies are well captured on a coarse grid.
\end{abstract}

Keywords: Navier-Stokes equations; Large eddy simulation; Turbulence; Rational LES model; Taylor LES model

\section{INTRODUCTION}

Large eddy simulation (LES) is currently one of the most promising approaches for the simulation of turbulent flows. In contrast to the direct simulation (DNS), it does not aim to resolve all length scales in the flow. Given a length scale $\delta$, which is often connected to the mesh size, LES seeks to compute all flow features of size $O(\delta)$ accurately. There are many approaches to develop LES models but broadly they can be divided into phenomenological models (such as the Smagorinsky model, 1963), models based on combination of phenomenology and extrapolation from the resolved scales (such as the dynamic model by Germano et al., 1991, and mixed scale similarity models) and models derived using formal but systematic asymptotics (such as RNG models and the models we consider herein). These different approaches are well surveyed in Sagaut (2001).

In this report, we compare two models derived via asymptotics in wave number space. The first LES model we consider evolved from works of Leonard (1974) and Clark et al. (1979). Since its derivation (given in "The LES Models and Their Derivation" section) is based on a truncated Taylor polynomial approximation in wave number space, we shall call this the "Taylor LES model".
It is also discussed in current publications, e.g. in Pope (2000, Chapter 13) or in Sagaut (2001). The second model's derivation (also reviewed in "The LES models and their derivation" section) follows a similar path but is based instead on a Padé approximation - giving the "rational LES model". We study two types of the rational LES model. We also include the Smagorinsky model in our comparison.

There are many LES models and many more reports of success with them on very complicated problems. Our goal is complementary to this work: we seek to find the simplest problem for which one model fails clearly while others do not. Thus, we hope to draw a clear distinction among models for a problem as simple as possible. To further focus this paper, we are considering primarily two models based upon a common idea of systematic asymptotic approximation in wave number space. Since we are also interested in flows driven by interaction of a fluid with a boundary, we have selected the driven cavity problem in 2D and 3D. Further comparisons using LES benchmark problems, e.g. Rodi et al. (1997), are very valuable and in progress. Numerical studies of the Taylor LES model for the 2D driven cavity problem have appeared, e.g. in Cantekin et al. (1994) and in Coletti (1998).

This report investigates primarily if the considered LES models fulfil or violate two simple necessary conditions

*Corresponding author. Tel.: +49-391-67-12633. Fax: +49-391-67-18073. E-mail: john@mathematik.uni-magdeburg.de 
which are, in our opinion, required for a useful LES model:

the LES model should replicate laminar flows,

the solution obtained with the LES model should have bounded total kinetic energy for high Reynolds number $(R e)$ flows.

Most LES studies adopt the complementary approach of checking if a model can be tuned to satisfy a sufficient condition (such as matching flow statistics) for a particular problem. Violation of one of the above two necessary conditions for a simple problem leads to a reliable conclusion that a model has serious defects. Of course, if both are satisfied it does not necessarily follow that the model is good. We shall see that even these two simple conditions allow us to draw a clear distinction among asymptotic models: the rational LES model fulfil both while the Taylor LES model fails the second one.

The rational LES model is compared further to the Smagorinsky model. An important drawback of the Smagorinsky model is that it introduces in general too much diffusion into the computed flow, i.e. the computed solution looks like a solution of a low Reynolds number flow, in particular, the main eddy in the driven cavity flow is too small. Our numerical tests show that the solutions obtained with both types of the rational LES model on a very coarse grid reproduce the main eddy of a high Reynolds number driven cavity flow much larger and centred more accurately than the Smagorinsky model. The LES computations were carried out on rather coarse grids which reflects the typical situation of turbulent flow computations of having too few degrees of freedom (d.o.f.) to resolve all flow structures.

The paper is organised as follows. The second section contains a brief derivation of the LES models, which are involved in the numerical study. The numerical tests are presented in the third section. The first part of this section is devoted to the study of the LES models in the 2D driven cavity problem at a low and at a high Reynolds number flow. The second part contains the numerical results of a high Reynolds number flow in the 3D driven cavity. The conclusions of the numerical studies are given in the fourth section.

\section{THE LES MODELS AND THEIR DERIVATION}

This section presents a traditional Taylor LES model introduced by Clark et al. (1979) and a new rational LES model, Galdi and Layton (2000), pointing out the essential difference in their derivation.

We consider an incompressible viscous fluid flowing in a bounded domain $\Omega$ in $\mathbb{R}^{d}, d=2,3$, and driven by body forces $\mathbf{f}$ or boundary velocities $\mathbf{g}$. In nondimensionalised terms, its velocity $\mathbf{u}$ and pressure $p$ are solutions of the Navier-Stokes equations (NSE) given by

$$
\begin{aligned}
\frac{\partial \mathbf{u}}{\partial t}-R e^{-1} \Delta \mathbf{u}+\mathbf{u} \cdot \nabla \mathbf{u}+\nabla p & =\mathbf{f} & & \text { in } \Omega \times(0, T], \\
\nabla \cdot \mathbf{u} & =0 & & \text { in } \Omega \times(0, T], \\
\mathbf{u}(\mathbf{x}, 0) & =\mathbf{u}_{0}(\mathbf{x}) & & \text { in } \Omega, \\
\mathbf{u} & =\mathbf{g} & & \text { on } \partial \Omega \times(0, T],
\end{aligned}
$$

where $\partial \Omega$ is the boundary of $\Omega$ with outer normal $\mathbf{n}$, $\int_{\Omega} p(\mathbf{x}) \mathrm{d} \mathbf{x}=0, \nabla \cdot \mathbf{u}_{0}=0$ and $\int_{\partial \Omega} \mathbf{g} \cdot \mathbf{n} \mathrm{d} s=0$.

LES models try to compute spatial averages of the velocity and the pressure. The spatial averages are obtained through convolution with a space filter. One common filter, which we select herein, is the Gaussian filter

$$
g_{\delta}(\mathbf{x}):=\left(\frac{\gamma}{\pi}\right)^{d / 2} \frac{1}{\delta^{d}} \exp \left(-\gamma \frac{\|\mathbf{x}\|_{2}^{2}}{\delta^{2}}\right),
$$

where $\gamma$ is a constant (often $\gamma=6$, and this is the value we are using herein), $\delta$ is the averaging radius and $\|\mathbf{x}\|_{2}$ the Euclidean norm of $\mathbf{x}$. Extending all variables appropriately outside $\Omega$, the convolution $\overline{\mathbf{u}}=g_{\delta} * \mathbf{u}$ represents the eddies of size $O(\delta)$ or larger.

Remark 2.1 The main property of the filter which defines $\overline{\mathbf{u}}$ is to suppress all flow structures smaller than $O(\delta)$. That means in Fourier space that the Fourier transform of the filter attenuates high frequencies. Figure 1 shows that the Fourier transform of the Gaussian filter almost vanishes for large wave number components, which leads to the strong damping of the high frequencies of the filtered function.

For constant filter width $\delta$ and points $\mathbf{x} \in \Omega$ sufficiently away from $\partial \Omega$, differentiation and convolution commute up to exponentially small terms. Thus, applying this

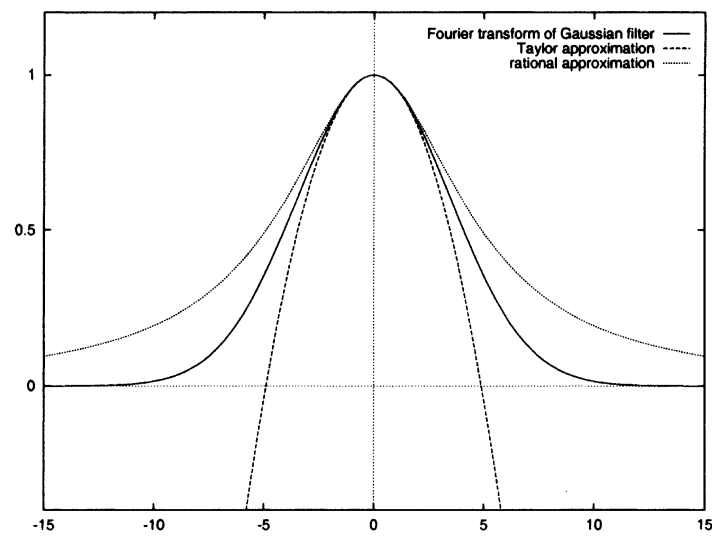

FIGURE 1 The Fourier transform of the Gaussian filter with Taylor and rational approximation. 
averaging operator to the Navier-Stokes equations gives the set of space-filtered Navier-Stokes equations

$$
\begin{array}{rlrl}
\frac{\partial \overline{\mathbf{u}}}{\partial t}-R e^{-1} \Delta \overline{\mathbf{u}} & & \\
+\nabla \cdot(\overline{\mathbf{u u}})+\nabla \bar{p} & =\overline{\mathbf{f}} & & \text { in } \Omega \times(0, T], \\
\nabla \cdot \overline{\mathbf{u}} & =0 & & \text { in } \Omega \times(0, T] \\
\overline{\mathbf{u}}(\mathbf{x}, 0) & =\overline{\mathbf{u}}_{0}(\mathbf{x}) & & \text { in } \Omega, \\
\overline{\mathbf{u}}(\mathbf{x}, t) & =\left(g_{\delta} * \mathbf{u}\right)(\mathbf{x}, t) & & \text { on } \partial \Omega \times(0, T] .
\end{array}
$$

To perform a LES, closure in Eq. (2) must be addressed to obtain a system whose solution approximates $(\overline{\mathbf{u}}, \bar{p})$. Various models of the stress tensor $\overline{\mathbf{u u}}$ are possible, e.g. see Aldama (1990) or Sagaut (2001). One way to derive models for $(\overline{\mathbf{u}}, \bar{p})$ uses the decomposition $\mathbf{u}=\overline{\mathbf{u}}+\mathbf{u}^{\prime}$, which gives

$$
\overline{\mathbf{u} \mathbf{u}}=\overline{\left(\overline{\mathbf{u}}+\mathbf{u}^{\prime}\right)\left(\overline{\mathbf{u}}+\mathbf{u}^{\prime}\right)}=\overline{\overline{\mathbf{u}}} \overline{\mathbf{u}}+\overline{\overline{\mathbf{u}} \mathbf{u}^{\prime}+\mathbf{u}^{\prime} \overline{\mathbf{u}}}+\overline{\mathbf{u}^{\prime} \mathbf{u}^{\prime}} .
$$

Equation (3) is a decomposition of the averaged nonlinear interactions into "resolved scales", $\overline{\overline{\mathbf{u}}} \overline{\mathbf{u}}$, "cross terms", $\overline{\overline{\mathbf{u}} \mathbf{u}^{\prime}+\mathbf{u}^{\prime} \overline{\mathbf{u}}}$, describing the interaction of large and small eddies, and the "subgrid scale" term $\overline{\mathbf{u}^{\prime} \mathbf{u}^{\prime}}$, describing the effects of the interaction of small eddies on the mean flow. It is well known (Germano, 1986, see also Pope, 2000; Sagaut, 2001) that the terms in Eq. (3) are not individually Galilean invariant. Thus, for phenomenological modelling, these should be redefined following Germano (1986). For asymptotic modelling, the procedure described below results in the same model with either regrouping.

The modelling technique used in the derivation of the class of LES models considered in this report employs a closure approximation via systematic asymptotics in Fourier space. The principal steps in this mathematically based approach are

1. apply the Fourier transform to each term of Eq. (3),

2. if necessary, replace the Fourier transform $\mathscr{F}\left(\mathbf{u}^{\prime}\right)$ by $\mathscr{F}(\overline{\mathbf{u}})$ using

$$
\mathscr{F}\left(\mathbf{u}^{\prime}\right)=\mathscr{F}(\mathbf{u}-\overline{\mathbf{u}})=\frac{\mathscr{F}(\overline{\mathbf{u}})}{\mathscr{F}\left(g_{\delta}\right)}-\mathscr{F}(\overline{\mathbf{u}}),
$$

3. approximate the Fourier transform of the Gaussian filter $\mathscr{F}\left(g_{\delta}\right)$ by a simpler function,

4. neglect all terms which are formally of order $\delta^{4}$,

5. apply the inverse Fourier transform and simplify.

This approach was used the first time by Leonard (1974). To illustrate it, consider for example the first term in Eq. (3). Step 1 gives

$$
\mathscr{F}(\overline{\overline{\mathbf{u}}} \overline{\mathbf{u}})=\mathscr{F}\left(g_{\delta}\right) \mathscr{F}(\overline{\mathbf{u}} \overline{\mathbf{u}})=\exp \left(-\frac{\delta^{2}}{4 \gamma}\|\mathbf{k}\|_{2}^{2}\right) \mathscr{F}(\overline{\mathbf{u}} \overline{\mathbf{u}}),
$$

where $\mathbf{k}$ is the $\mathbf{k}$-Fourier wave number. The Taylor LES model, see e.g. Leonard (1974) and Clark et al. (1979), uses in step 3 the Taylor polynomial approximation

$$
\exp \left(-\frac{\delta^{2}}{4 \gamma}\|\mathbf{k}\|_{2}^{2}\right)=1-\frac{\|\mathbf{k}\|_{2}^{2}}{4 \gamma} \delta^{2}+O\left(\delta^{4}\right),
$$

see Fig. 1, leading to

$$
\begin{aligned}
\mathscr{F}(\overline{\overline{\mathbf{u}}} \overline{\mathbf{u}}) & =\left(1-\frac{\|\mathbf{k}\|_{2}^{2}}{4 \gamma} \delta^{2}\right) \mathscr{F}(\overline{\mathbf{u}} \overline{\mathbf{u}})+C \delta^{4} \mathscr{F}(\overline{\mathbf{u}} \overline{\mathbf{u}}) \\
& \approx\left(1-\frac{\|\mathbf{k}\|_{2}^{2}}{4 \gamma} \delta^{2}\right) \mathscr{F}(\overline{\mathbf{u}} \overline{\mathbf{u}}) .
\end{aligned}
$$

Note, the dropped term depends on $\overline{\mathbf{u}}$ and thus there is a dependency on $\delta$ beside the factor $\delta^{4}$. That means, we have only a formal asymptotic expansion. Applying the inverse Fourier transform gives the model

$$
\overline{\overline{\mathbf{u}}} \overline{\mathbf{u}} \approx \overline{\mathbf{u}} \overline{\mathbf{u}}+\frac{\delta^{2}}{4 \gamma} \Delta(\overline{\mathbf{u}} \overline{\mathbf{u}}) .
$$

Thus, the resolved scales have the formal order $\overline{\overline{\mathbf{u}}} \overline{\mathbf{u}}=$ $O(1)$. A similar formal estimate of the other orders of magnitudes gives

$$
\overline{\overline{\mathbf{u}}} \overline{\mathbf{u}}=O(1), \quad \overline{\overline{\mathbf{u}} \mathbf{u}^{\prime}+\mathbf{u}^{\prime} \overline{\mathbf{u}}}=O\left(\delta^{2}\right), \quad \overline{\mathbf{u}^{\prime} \mathbf{u}^{\prime}}=O\left(\delta^{4}\right) .
$$

Applying step 1 through 5 also to the other terms in Eq. (3) using the Taylor polynomial approximation given in Eq. (4) gives the Taylor LES model

$$
\begin{array}{rlrl}
\frac{\partial \mathbf{w}}{\partial t}-R e^{-1} \Delta \mathbf{w}+\nabla \cdot(\mathbf{w w})+\nabla q & & \\
+\nabla \cdot\left(\frac{\delta^{2}}{2 \gamma} \nabla \mathbf{w} \nabla \mathbf{w}\right) & =\overline{\mathbf{f}} & & \text { in } \Omega \times(0, T], \\
\nabla \cdot \mathbf{w} & =0 & & \text { in } \Omega \times(0, T], \\
\mathbf{w}(\mathbf{x}, 0) & =\overline{\mathbf{u}}_{0}(\mathbf{x}) & & \text { in } \Omega, \\
\mathbf{w}(\mathbf{x}, t) & =\left(g_{\left.\delta^{*} \mathbf{u}\right)(\mathbf{x}, t)}\right. & & \text { on } \partial \Omega \times(0, T],
\end{array}
$$

where $(\mathbf{w}, q)$ is an approximation to $(\overline{\mathbf{u}}, \bar{p})$ and $(\nabla \mathbf{w} \nabla \mathbf{w})_{i, j}$ is shorthand for

$$
\sum_{l} \frac{\partial \mathbf{w}_{i}}{\partial \mathbf{x}_{l}} \frac{\partial \mathbf{w}_{j}}{\partial \mathbf{x}_{l}}
$$

The turbulent fluctuations $\overline{\mathbf{u}^{\prime} \mathbf{u}^{\prime}}$ in Eq. (3) are modelled in this approach by zero because they are formally $O\left(\delta^{4}\right)$, Eq. (5). Therefore, they are neglected in step 4 of the general approach. However, $\overline{\mathbf{u}^{\prime} \mathbf{u}^{\prime}}$ is believed to represent an important physical mechanism in turbulence. The usual way to model $\overline{\mathbf{u}^{\prime} \mathbf{u}^{\prime}}$, e.g. used by Aldama (1990) or 
Coletti (1998), is by a Smagorinsky (1963) term of the form

$$
C_{\mathrm{s}} \delta^{2}\|\mathbb{D}(\overline{\mathbf{u}})\| \mathbb{D}(\overline{\mathbf{u}})
$$

which is added to the left hand side of Eq. (6). Here $\mathbb{D}(\overline{\mathbf{u}})=\left(d_{i j}\right)_{i, j=1, \ldots, d}:=\left(\nabla \overline{\mathbf{u}}+(\nabla \overline{\mathbf{u}})^{T}\right) / 2$ is the deformation tensor associated with $\overline{\mathbf{u}}$ and

$$
\|\mathbb{D}(\overline{\mathbf{u}})\|=\left(\sum_{i, j=1}^{d} d_{i j}^{2}\right)^{1 / 2} .
$$

Thus, the Taylor LES model becomes

$$
\begin{array}{rlrl}
\frac{\partial \mathbf{w}}{\partial t}-R e^{-1} \Delta \mathbf{w}+\nabla \cdot(\mathbf{w w})+\nabla q & & \\
+\nabla \cdot\left(\frac{\delta^{2}}{2 \gamma} \nabla \mathbf{w} \nabla \mathbf{w}\right)-\nabla \cdot\left(C_{\mathrm{s}} \delta^{2}\right. & & \\
\times\|\mathbb{D}(\mathbf{w})\| \mathbb{D}(\mathbf{w})) & =\overline{\mathbf{f}} & & \text { in } \Omega \times(0, T], \\
\nabla \cdot \mathbf{w} & =0 & & \text { in } \Omega \times(0, T], \\
\mathbf{w}(\mathbf{x}, 0) & =\overline{\mathbf{u}}_{0}(\mathbf{x}) & & \text { in } \Omega, \\
\mathbf{w}(\mathbf{x}, t) & =\left(g_{\delta} * \mathbf{u}\right)(\mathbf{x}, t) & & \text { on } \partial \Omega \times(0, T] .
\end{array}
$$

Existence and uniqueness of a weak solution of Eq. (8) is proved by Coletti (1998) following the analysis by Ladyzhenskaya (1967). This theory has one pivotal condition: the constant $C_{\mathrm{s}}$ has to be large enough such that Smagorinsky term given in Eq. (7) for $\overline{\mathbf{u}^{\prime} \mathbf{u}^{\prime}}$ dominates the term $\left(\delta^{2} / 2 \gamma\right) \nabla \mathbf{w} \nabla \mathbf{w}$ arising essentially in modelling the cross terms. This technical condition is questionable since $\overline{\mathbf{u}^{\prime} \mathbf{u}^{\prime}}=O\left(\delta^{4}\right)$ while the cross terms are $O\left(\delta^{2}\right)$, Eq. (5).

The Taylor polynomial approximation in Eq. (4) used in the derivation of model (8) violates the most important property of the Fourier transform of a filter function namely that it (almost) vanishes for high frequencies, see Remark 2.1. In contrast, the absolute values of the Taylor polynomial approximation become even larger the higher the frequency is, see Fig. 1. Thus, the high frequencies are stimulated rather than attenuated. This incorrect stimulation of high frequencies plays an important role in numerical calculations at high Reynolds numbers, since increasing the high wave number components (large $\|\mathbf{k}\|_{2}$ ) in the Fourier space is equivalent to increasing the small scales (small $\|\mathbf{x}\|_{2}$ ) in the physical space $\left(\mathbb{R}^{d}\right)$. We believe that this is the true cause of the need for powerful dissipative mechanisms, such as the $O\left(\delta^{2}\right)$ Smagorinsky model for $\overline{\mathbf{u}^{\prime} \mathbf{u}^{\prime}}$.

Motivated by the above observation, in Galdi and Layton (2000) a modified model was developed, which is consistent with the required attenuation of high frequencies in $\overline{\mathbf{u}}$. This model is based upon repeating steps 1 through 5 above using a rational approximation of $\mathscr{F}\left(g_{\delta}\right)$, the $(0,1)$ Padé approximation,

$$
\mathscr{F}\left(g_{\delta}\right)=\exp \left(-\frac{\delta^{2}}{4 \gamma}\|\mathbf{k}\|_{2}^{2}\right)=\frac{1}{1+\frac{\delta^{2}}{4 \gamma}\|\mathbf{k}\|_{2}^{2}}+O\left(\delta^{4}\right)
$$

see also Fig. 1, instead of a Taylor expansion. It is possible to approximate $\mathscr{F}\left(g_{\delta}\right)$ by Eq. (9) in all places or only in some places in step 3 of the general approach leading to two variants of the rational LES model. The model through $O\left(\delta^{2}\right)$ terms is

$$
\begin{aligned}
\frac{\partial \mathbf{w}}{\partial t}-R e^{-1} \Delta \mathbf{w}+\nabla \cdot(\mathbf{w w})+ & \nabla q & & \\
+\nabla \cdot\left[A\left(\frac{\delta^{2}}{2 \gamma} \nabla \mathbf{w} \nabla \mathbf{w}\right)\right] & =\overline{\mathbf{f}} & & \text { in } \Omega \times(0, T], \\
\nabla \cdot \mathbf{w} & =0 & & \text { in } \Omega \times(0, T], \\
\mathbf{w}(\mathbf{x}, 0) & =\overline{\mathbf{u}}_{0}(\mathbf{x}) & & \text { in } \Omega, \\
\mathbf{w}(\mathbf{x}, t) & =\left(g_{\delta} * \mathbf{u}\right)(\mathbf{x}, t) & & \text { on } \partial \Omega \times(0, T],
\end{aligned}
$$

where, depending on the operator $A$, two types of the rational LES model can be distinguished: 1. $A=\left(-\frac{\delta^{2}}{4 \gamma} \Delta+I\right)^{-1}$-rational LES model with auxiliary
problem,

2. $A=g_{\delta}{ }^{*}$-rational LES model with convolution.

The auxiliary problem can be considered as an approximation of the convolution operator.

The subgrid scale term $\overline{\mathbf{u}^{\prime} \mathbf{u}^{\prime}}$ is formally $O\left(\delta^{4}\right)$, hence, can be formally neglected. Since our goal is to do a careful comparison between the Taylor and the rational models, we will perform experiments including the same Smagorinsky term given in Eq. (7) as a model for $\overline{\mathbf{u}^{\prime} \mathbf{u}^{\prime}}$ for both. These are supplemented with experiments for the rational LES model without Smagorinsky term. Other proposals of modelling $\overline{\mathbf{u}^{\prime} \mathbf{u}^{\prime}}$ are given in Iliescu and Layton (1998).

The rational LES model with auxiliary problem we consider is

$$
\begin{array}{rlrl}
\frac{\partial \mathbf{w}}{\partial t}-R e^{-1} \Delta \mathbf{w}+\nabla \cdot(\mathbf{w w})+\nabla q & & \\
+\nabla \cdot\left[\left(-\frac{\delta^{2}}{4 \gamma} \Delta+I\right)^{-1}\left(\frac{\delta^{2}}{2 \gamma} \nabla \mathbf{w} \nabla \mathbf{w}\right)\right]-\nabla \cdot\left(C_{\mathrm{s}} \delta^{2}\right. \\
\times\|\mathbb{D}(\mathbf{w})\| \mathbb{D}(\mathbf{w}))=\overline{\mathbf{f}} & & \text { in } \Omega \times(0, T], \\
\nabla \cdot \mathbf{w} & =0 & & \text { in } \Omega \times(0, T], \\
\mathbf{w}(\mathbf{x}, 0) & =\overline{\mathbf{u}}_{0}(\mathbf{x}) & & \text { in } \Omega, \\
\mathbf{w}(\mathbf{x}, t) & =\left(g_{\delta} * \mathbf{u}\right)(\mathbf{x}, t) & & \text { on } \partial \Omega \times(0, T] .
\end{array}
$$

We have used homogeneous Neumann boundary conditions for the auxiliary problem as suggested in Galdi and Layton (2000). 
In the rational LES model with convolution, the first equation of Eq. (10) is replaced by

$$
\begin{aligned}
\frac{\partial \mathbf{w}}{\partial t} & -R^{-1} \Delta \mathbf{w}+\nabla \cdot(\mathbf{w w})+\nabla q \\
+ & \nabla \cdot\left[g_{\delta^{*}}\left(\frac{\delta^{2}}{2 \gamma} \nabla \mathbf{w} \nabla \mathbf{w}\right)\right] \\
& -\nabla \cdot\left(C_{\mathrm{s}} \delta^{2}\|\mathbb{D}(\mathbf{w})\| \mathbb{D}(\mathbf{w})\right)=\overline{\mathbf{f}} \quad \text { in } \Omega \times(0, T],
\end{aligned}
$$

where the convolution is approximated numerically. All functions which are convolved are continued by zero outside $\Omega$.

Since there is a lot of computational experience with the Smagorinsky model

$$
\begin{array}{rlrl}
\frac{\partial \mathbf{w}}{\partial t}-R e^{-1} \Delta \mathbf{w}+\nabla \cdot(\mathbf{w w})+\nabla q-\nabla \cdot\left(C_{\mathrm{s}} \delta^{2}\right. \\
\times\|\mathbb{D}(\mathbf{w})\| \mathbb{D}(\mathbf{w}))=\overline{\mathbf{f}} & & \text { in } \Omega \times(0, T], \\
\nabla \cdot \mathbf{w} & =0 & & \text { in } \Omega \times(0, T], \\
\mathbf{w}(\mathbf{x}, 0) & =\overline{\mathbf{u}}_{0}(\mathbf{x}) & & \text { in } \Omega, \\
\mathbf{w}(\mathbf{x}, t) & =\left(g_{\delta} * \mathbf{u}\right)(\mathbf{x}, t) & & \text { on } \partial \Omega \times(0, T],
\end{array}
$$

we have included tests with it as well. Although its drawbacks are well understood, this model is still popular due to its simplicity.

\section{THE NUMERICAL STUDIES}

Based on "The LES models and their derivation" section, the usual Galerkin finite element method for the NavierStokes equations (1), the Taylor LES model (8), the two variants of the rational LES model (10), (11) and the Smagorinsky model (12) are compared with respect to the criteria given in the introduction using the two- and three-dimensional driven cavity problem. The total kinetic energy of the computed velocity $\mathbf{w}^{h}$ is defined by

$$
E_{\mathrm{kin}}\left(\mathbf{w}^{h}\right):=\frac{1}{2} \int_{\Omega} \mathbf{w}^{h} \cdot \mathbf{w}^{h} \mathrm{~d} \mathbf{x} .
$$

\section{The Two-dimensional Driven Cavity Problem}

This problem is given by $\Omega=(0,1)^{2}$, the boundary conditions $\mathbf{w}=(1,0)$ for $0<x<1, y=1$, homogeneous Dirichlet boundary conditions at the other boundaries, and $\mathbf{f}=\mathbf{0}$. Although less popular than channel flow, it has been used in tests of LES models, including the Taylor LES model (see Cantekin et al., 1994; Coletti, 1998) and the dynamic subgrid scale model, (Zang et al., 1993). An appropriate initial condition for the time dependent problem is not agreed upon in the literature. For all models, we have tried to use a, so-called, impulsive start in our computations, i.e. the velocity inside the domain is chosen zero at the beginning. Investigations by Gresho and Sani (1998; 2000) show that an impulsive start can create a quickly decaying but nonphysical transient in the solution of an incompressible flow problem. For this reason, we repeated the tests which failed the boundedness criterion of the total kinetic energy with the impulsive start also with other initial conditions to make sure that their failure is not caused by the impulsive start.

We performed computations for a low Reynolds number flow $(R e=400)$ and a high Reynolds number flow $(R e=$ 10,000). All LES models are applied without turbulent viscosity for $R e=400\left(C_{\mathrm{s}}=0\right.$ in Eq. (7)) and with $C_{\mathrm{s}}=$ 0.01 in the tests for $R e=10,000$. The Smagorinsky model (12) was applied also with $C_{\mathrm{s}}=0.01$. Thus, $C_{\mathrm{s}}$ is small compared to most computations with the Smagorinsky model from the literature which use $C_{\mathrm{s}}>0.01$.

First, the LES models are discretised in time by the fractional-step- $\theta$-scheme, e.g. see Bristeau et al. (1987), which is analysed for the time dependent Navier-Stokes equations by Klouček and Rys (1994). This implicit scheme is of second order accuracy, more stable than the Crank-Nicolson scheme and is currently considered "best" on the basis of accuracy and reliability, e.g. see Turek (1999) or Rannacher (2000). Unless mentioned otherwise, all computations were carried out with the equidistant time step $\Delta t=0.01$.

The equations in each time step are linearised by a fixed point iteration and the linear equations arising in this iteration are discretised by the $Q_{2} / P_{1}^{\text {disc }}$ finite element (called $Q_{2} / P_{-1}$ in Gresho and Sani, 2000), i.e. the velocity is approximated by continuous piecewise biquadratics and the pressure by discontinuous linears. This conforming pair of finite element spaces fulfils the inf-sup or Babuška-Brezzi stability condition, (Matthies and Tobiska, 2002). It is currently considered the most stable and best performing element for finite element discretisations of Navier-Stokes equations, e.g. see Fortin (1993) or Gresho and Sani (2000). In addition, it has been proven to be superior to other pairs of finite elements in recent studies of benchmark problems for laminar flows (John and Matthies, 2001; John, 2002). All computations were carried out on equidistant grids with squares of size $h \times h$. We used the deformation tensor formulation

$$
2(\mathbb{D}(\mathbf{w}), \mathbb{D}(\mathbf{v}))
$$

as variational form of the viscous term. These choices give a discretisation which is strictly conservative, so any blow up or excessive smearing is caused by the model rather than the discretisation.

The fractional-step- $\theta$-scheme divides each time step into three sub time steps. In each sub time step, a nonlinear saddle point problem has to be solved. All terms in the Galerkin finite element method of the Navier-Stokes equations and of the Smagorinsky model are treated 
TABLE I 2D driven cavity problem, mesh widths and d.o.f.

\begin{tabular}{lccr}
\hline Mesh width & Velocity d.o.f. & Pressure d.o.f. & Total d.o.f. \\
\hline $1 / 16$ & 2178 & 768 & 2946 \\
$1 / 32$ & 8450 & 3072 & 11,522 \\
$1 / 64$ & 33,282 & 12,288 & 45,570 \\
\hline
\end{tabular}

always fully nonlinear. In the LES models, the convective term and the Smagorinsky term (7) are also treated implicitly, whereas the additional term coming from the LES models is computed in each sub time step before the nonlinear iteration and it is not changed within this iteration. The linear problems are solved by a coupled multigrid method with Vanka-type smoothers as studied numerically, e.g. in John and Tobiska (2000) and John and Matthies (2001) or John (2002).

We present results for the mesh widths $h=1 / 16, h=$ $1 / 32$ and $h=1 / 64$. The corresponding numbers of d.o.f. in space are given in Table I. If not mentioned otherwise, the averaging radius $\delta$ is chosen to be the diameter of the mesh cell, i.e. the longest distance of two of its vertices, which is $\delta=\sqrt{2} h$.

All computations were carried out with the code MooNMD (John and Matthies, 2002).

\section{The Low Reynolds Number Flow}

One property of a good LES model is that its use in a laminar or low Reynolds number flow results in a solution which is very close to the solution obtained by solving the Navier-Stokes equations. All models in our study show a very similar behaviour with respect to the total kinetic energy of the computed solutions, see Table II. Also the total kinetic energy of the differences to the solution obtained with the Galerkin FEM of the Navier-Stokes equation are small, compare Table III.

The behaviour of the Taylor LES model is not consistent with results in Coletti (1998). In Coletti (1998), the Taylor LES model does not give a satisfactory solution for the driven cavity problem with $R e=400$ and the $P_{2} / P_{1}$ spatial finite element discretisation (Taylor/ Hood) on a triangular mesh. Since the most essential difference between both studies are the finite element spaces, the $Q_{2} / P_{1}^{\text {disc }}$ element seems to have a better stabilisation effect on the Taylor LES model than the $P_{2} / P_{1}$ finite element.

TABLE II 2D driven cavity problem, total kinetic energy for the stationary state, $R e=400$

\begin{tabular}{lccc}
\hline Model & $h=1 / 16$ & $h=1 / 32$ & $h=1 / 64$ \\
\hline $\begin{array}{l}\text { Galerkin FEM } \\
\text { of the NSE }\end{array}$ & $4.222 \times 10^{-2}$ & $4.093 \times 10^{-2}$ & $4.083 \times 10^{-2}$ \\
$\begin{array}{l}\text { Taylor LES model } \\
\text { Rational LES with } \\
\quad \text { auxiliary problem }\end{array}$ & $\begin{array}{l}4.645 \times 10^{-2} \\
5.362 \times 10^{-2}\end{array}$ & $4.337 \times 10^{-2}$ & $4.162 \times 10^{-2}$ \\
$\begin{array}{l}\text { Rational LES with } \\
\text { convolution }\end{array}$ & $5.411 \times 10^{-2}$ & $4.378 \times 10^{-2}$ & $4.156 \times 10^{-2}$ \\
& & & $4.160 \times 10^{-2}$ \\
\hline
\end{tabular}

TABLE III 2D driven cavity problem, total kinetic energy of the difference $\left|E_{\mathrm{kin}}\left(\mathbf{w}^{h}-\mathbf{w}_{\mathrm{NSE}}^{h}\right)\right|$ between the LES solution and the Galerkin FEM solution $\mathbf{w}_{\mathrm{NSE}}^{h}$ of the Navier-Stokes equations, $R e=400$

\begin{tabular}{lccc} 
Model & $h=1 / 16$ & $h=1 / 32$ & $h=1 / 64$ \\
\hline Taylor LES model & $1.189 \times 10^{-3}$ & $1.669 \times 10^{-4}$ & $1.468 \times 10^{-5}$ \\
Rational LES with & $1.287 \times 10^{-3}$ & $9.730 \times 10^{-5}$ & $1.741 \times 10^{-6}$ \\
$\quad$ auxiliary problem & & & \\
$\begin{array}{c}\text { Rational LES with } \\
\text { convolution }\end{array}$ & $1.602 \times 10^{-3}$ & $1.242 \times 10^{-4}$ & $1.058 \times 10^{-5}$ \\
\hline
\end{tabular}

Note that all three LES models replicate well the low Reynolds number laminar flow.

\section{The High Reynolds Number Flow}

\section{The Blow up OF THE TAYlor LES Model}

Simulations with the Taylor LES model with Smagorinsky type turbulent viscosity of form (7) blow up in finite time for $R e=10,000$, see Figs. 2-4, 7 and 8 . Table IV shows that a refinement of the spatial mesh does not prevent the blow up. A reduction of the time step on the same mesh level prevents the blow up but on the next finer mesh level the model blows up again. The scalings in Table IV show that this does not occur as a function only of $\Delta t / \Delta h$, i.e. the blow up is not related to a simple CFL-type condition. When solving the driven cavity with an impulsive start, Gresho and Sani (1998) have shown that there is an initial, nonphysical transient that makes small time approximate solutions questionable. This small time transient is not responsible for the blow up behaviour because the same behaviour was observed when starting instead with fully developed flows as initial condition. Indeed, when we used the solutions of the other models for $h=1 / 64$ at $T=1000$ as initial conditions, see right lower picture in Fig. 5 for the solution obtained with the Galerkin FEM of the Navier-Stokes equations, the Taylor LES model blew up within only few time steps, see Figs. 2 and 3.

The failure of the Taylor LES model is consistent with results reported by Cantekin et al. (1994) and

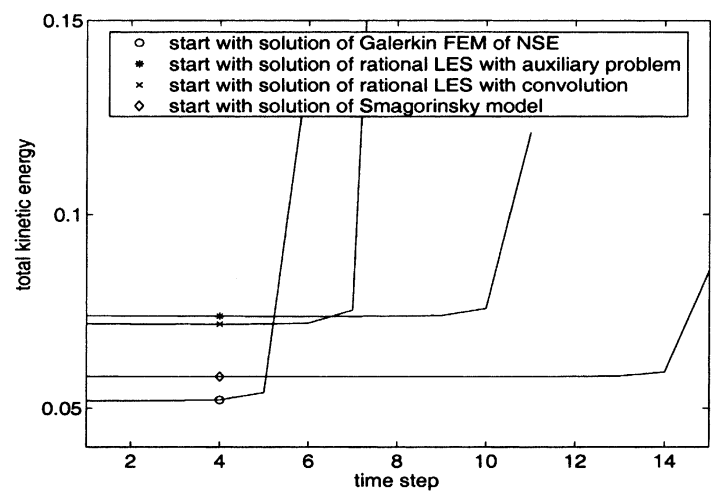

FIGURE 2 2D driven cavity problem, blow up of the Taylor LES model starting with various fully developed flow fields as starting conditions at $t=1000, h=1 / 64, \delta=\sqrt{2} / 64, \operatorname{Re}=10,000$. Note that the blow up does not depend on using an impulsive start. 


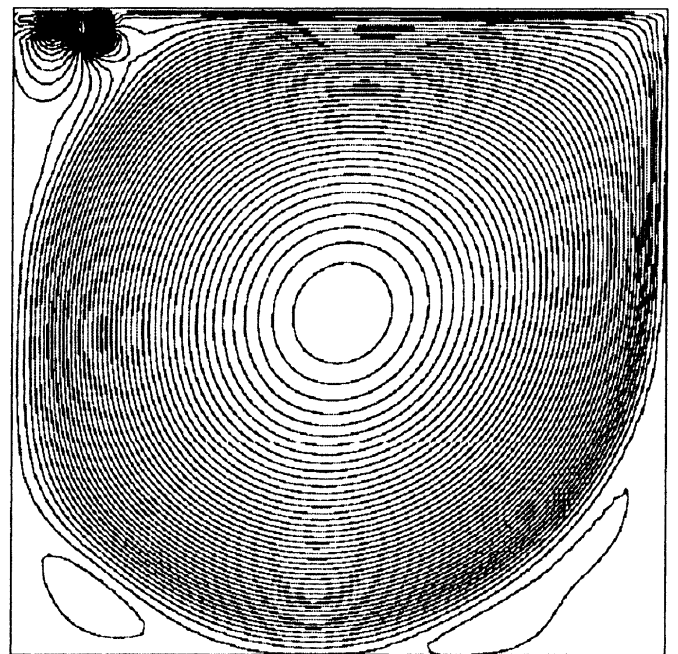

FIGURE 3 2D driven cavity problem, streamlines of the Taylor LES model solution just before blow up (time step 15 starting with the Smagorinsky model solution).

Coletti (1998). In Cantekin et al. (1994), a non-physical eddy near the left upper corner of the cavity is observed at Reynolds number $R e=3200$. The reasons of the appearance of this eddy, called "counter gradient diffusion", are discussed in Cantekin et al. (1994, p. 507 f.). It is interesting to observe that for Reynolds number $R e=$ 10,000 , the blow up of the solution starts at the position of the non-physical eddy, see Fig. 3. Coletti reports blow up of this model for $R e=10,000$ and the $P_{2} / P_{1}$ finite element discretisation. In other calculations, we have observed the same for the Taylor/Hood finite element on a quadrilateral grid $\left(Q_{2} / Q_{1}\right)$. Thus, it seems clear that the Taylor LES model does indeed stimulate rather than attenuate small eddies and it is not suited for turbulent flow computations.

\section{The Behaviour of the Rational LES Model}

For the rational LES model with the auxiliary problem and the rational LES model with convolution, both with turbulent viscosity of type (7), the total kinetic energy stays bounded for all parameters in contrast to the Taylor LES model, see Figs. 4, 7 and 8. Both variants of the rational LES model give similar results for the total kinetic energy in all tests.

The computations for the mesh width $h=1 / 16$ reflect best the normal condition for the numerical simulation of turbulent flows which is a very coarse mesh compared to the size of the small flow structures. The result in Fig. 4 shows the blow up of the Galerkin FEM of the NavierStokes equations and the Taylor LES model. A comparison of the computed flow fields with the flow field of the Galerkin FEM of the Navier-Stokes equations on a much finer grid, Fig. 5, shows that the solutions of the rational LES model are reasonable, whereas the Smagorinsky model is overly diffusive. The value of the
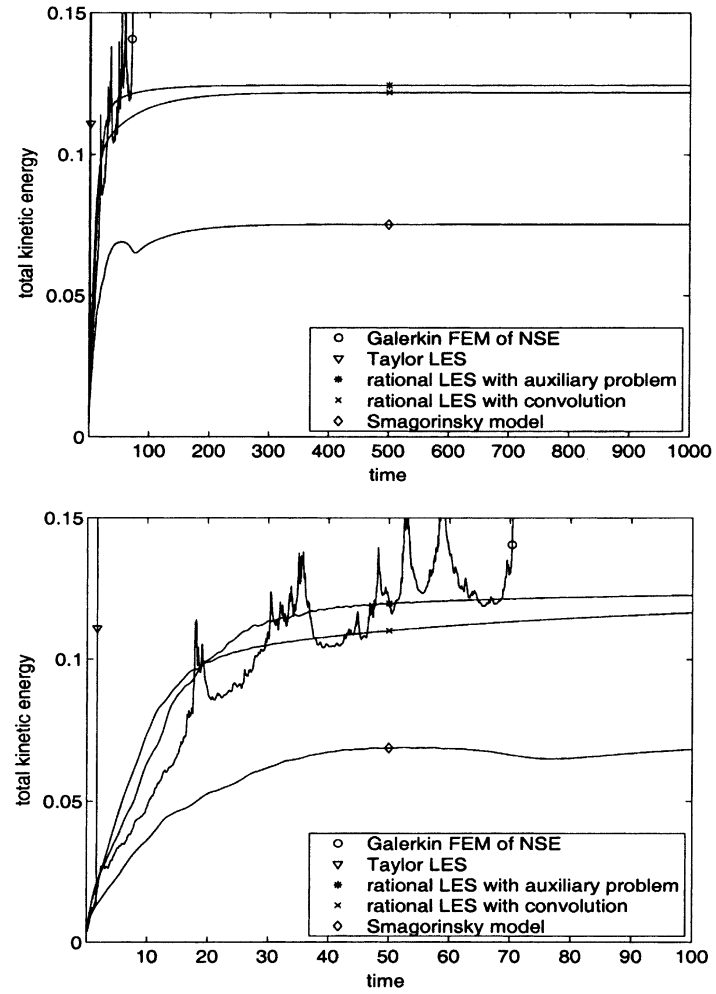

FIGURE 4 2D driven cavity problem, total kinetic energy, $R e=$ $10,000, h=1 / 16, \delta=\sqrt{2} / 16$.

averaging radius in these computations is $\delta=\sqrt{2} / 16 \approx$ 0.09 . Equilibrium driven cavity DNS simulations include smaller subeddies in the corners (Ghia et al., 1982). Our time dependent simulation of averages of $\mathbf{u}$ do not show these. This is correct and expected: all flow structures of size 0.09 or smaller should not be seen correctly in the computed solutions. This explains the fact that some of the sub-eddies in the corners, which can be seen in reference solutions, (Ghia et al., 1982), are not resolved accurately. The only eddy which should be computed accurately for this value of $\delta$ is the main centre eddy. This is done much better, as expected, by both types of the rational LES model than the Smagorinsky model.

The total kinetic energy of the solutions obtained with the Galerkin FEM of the Navier-Stokes equations is considerably different for $h=1 / 32$ and $h=1 / 64$.

TABLE IV 2D driven cavity problem, blow up times for the Taylor LES model, $R e=10,000, d=\sqrt{2} / 64$

\begin{tabular}{lrr}
\hline$\Delta t$ & $h=1 / 64$ & $h=1 / 128$ \\
\hline $10^{-1}$ & $2 \times 10^{-1}$ & $10^{-1}$ \\
$10^{-2}$ & $19 \times 10^{-2}$ & $2 \times 10^{-2}$ \\
$10^{-3}$ & no blow up & $8 \times 10^{-3}$ \\
$10^{-4}$ & no blow up & $239 \times 10^{-4}$ \\
\hline
\end{tabular}

Note that the blow up behaviour changes if $\delta$ is fixed and $\Delta t$ and $h$ are reduced. 

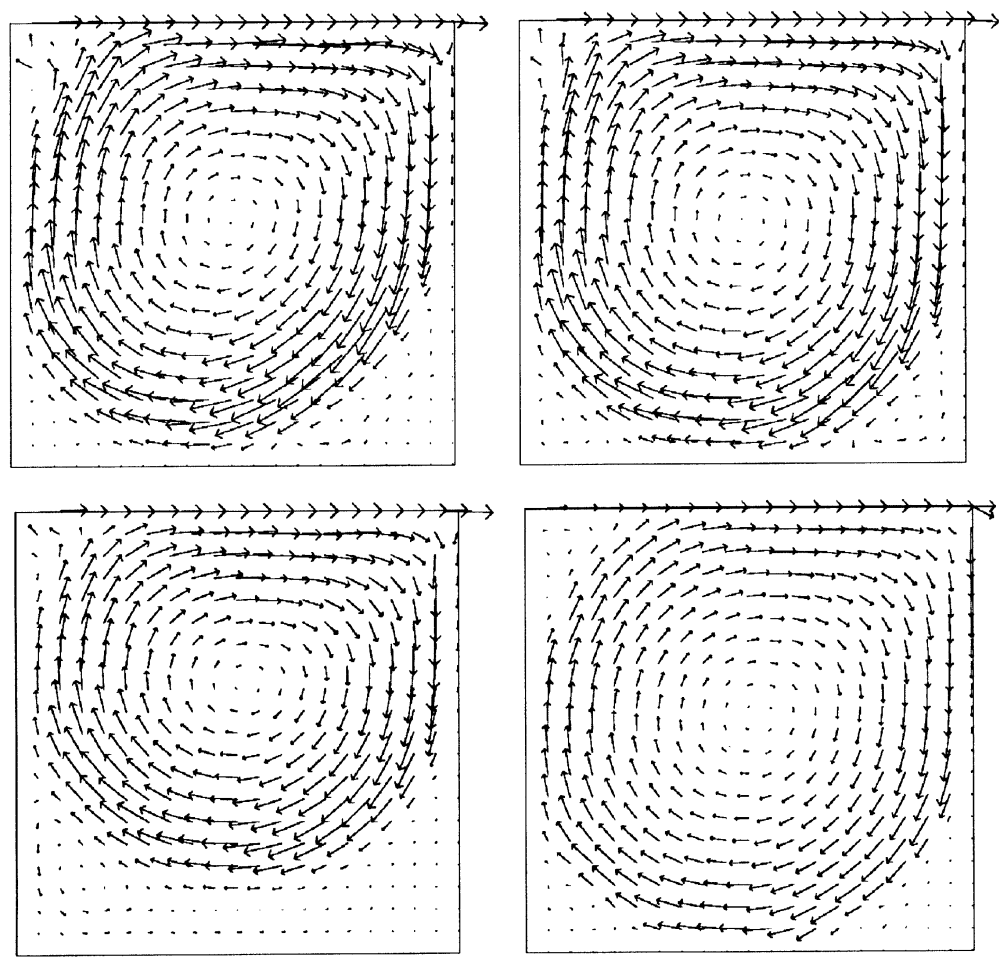

FIGURE 5 2D driven cavity problem, velocity for $R e=10,000$ and $t=1000$ : upper left: rational LES model with auxiliary problem $(h=1 / 16$, $\delta=\sqrt{2} / 16)$, upper right: rational LES model with convolution $(h=1 / 16, \delta=\sqrt{2} / 16)$, lower left: Smagorinsky model $(h=1 / 16, \delta=\sqrt{2} / 16)$, lower right: Galerkin FEM of the Navier-Stokes equations $(h=1 / 64)$. Note that both variants of the rational LES model reproduce the main eddy well, whereas the main eddy of the solution of the Smagorinsky model is too small and its centre is too close to the upper lid.

This indicates that the discretisation error in space is still relatively large. The total kinetic energy of the discrete solutions in all models decreases for finer meshes. For $h=1 / 32$, the total kinetic energy of the solution with the Galerkin FEM of the Navier-Stokes equations is larger than for the solution of the Smagorinsky model whereas the situation for $h=1 / 64$ is vice versa. The total kinetic energy of the solutions for both types of the rational LES model was always somewhat larger than for the solutions of the Galerkin FEM of the Navier-Stokes equations and the Smagorinsky model.

Numerical tests with the rational LES model without Smagorinsky term, $C_{\mathrm{s}}=0$, show a blow up of the solutions. The results presented in Fig. 6 were started with the solutions of the computations with the Smagorinsky term. The failing of the rational LES model without Smagorinsky term is not completely understood, but suggests that including a model of the effects of the turbulent fluctuations $\overline{\mathbf{u}^{\prime} \mathbf{u}^{\prime}}$ is important.

\section{The Three-dimensional Driven Cavity Problem}

The three-dimensional driven cavity problem is defined in the unit cube $(0,1)^{3}$. The boundary condition $\mathbf{w}=(1,0,0)$ is prescribed at the upper lid of the cavity $0<x<1$, $0<y<1, z=1$. At the other boundaries, homogeneous Dirichlet boundary conditions are applied. The right hand side of the momentum equation vanishes in $\Omega, \mathbf{f}=\mathbf{0}$.

We used the fractional-step- $\theta$-scheme as temporal discretisation and the $Q_{2} / P_{1}^{\text {disc }}$ finite element as spatial discretisation. We present results for the Reynolds

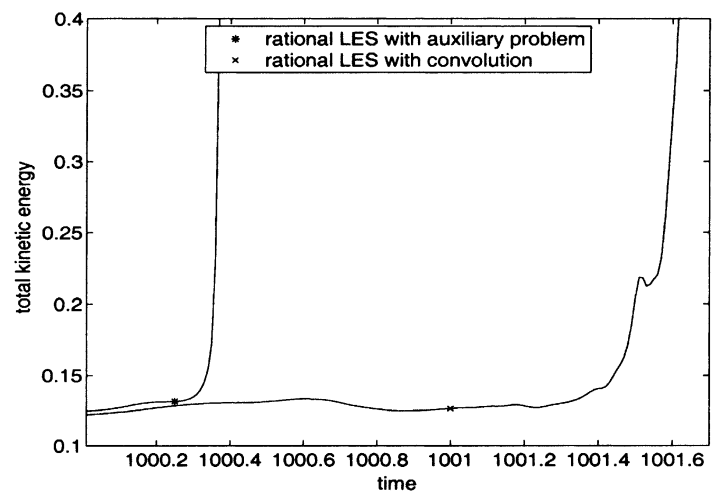

FIGURE 6 2D driven cavity problem, blow up of the total kinetic energy in the rational LES model without Smagorinsky term, $R e=$ $10,000, h=1 / 16, \delta=\sqrt{2} / 16$. 

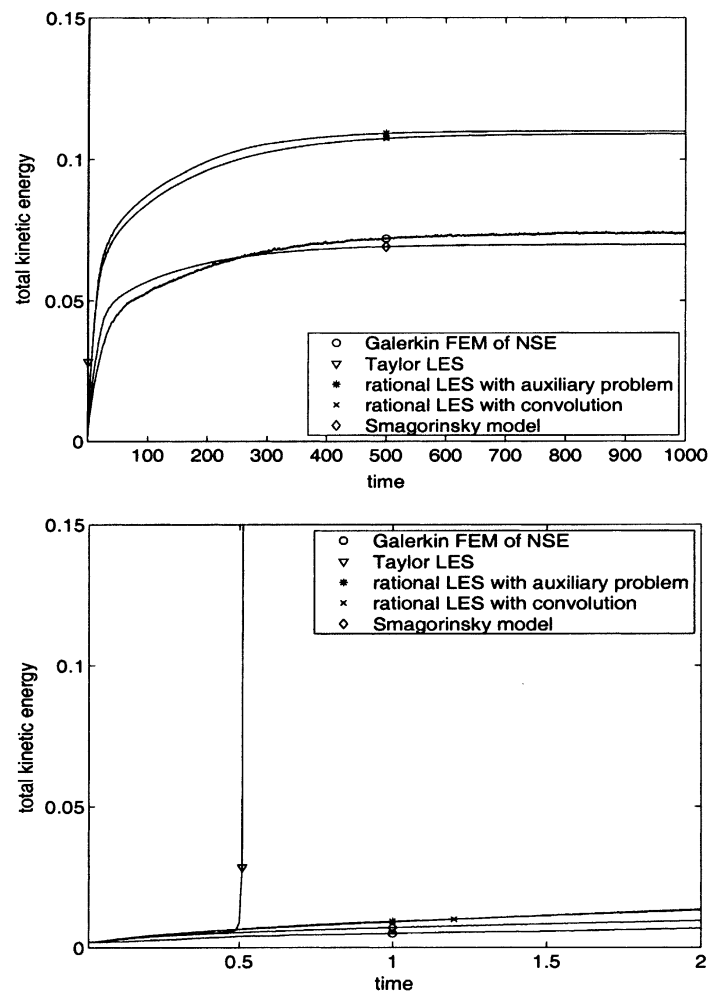

FIGURE 7 2D driven cavity problem, total kinetic energy, $R e=$ $10,000, h=1 / 32, \delta=\sqrt{2} / 32$. Note the blow up of the Taylor LES model.

number $R e=10,000$ on a $h \times h \times h$ mesh with $h=$ $1 / 8$. This mesh width leads to 14,739 d.o.f. of the velocity and 2048 d.o.f of the pressure. The following parameters are used in the computations:

$$
\begin{aligned}
& \delta=\sqrt{3} / 8 \text { (diagonals of the mesh cells), } \\
& C_{\mathrm{s}}=0.01 \\
& \Delta t=0.1
\end{aligned}
$$

impulsive start.

The total kinetic energy of the computed solutions is presented in Fig. 9. The observations are similar as in the two-dimensional driven cavity problem. The Galerkin finite element discretisation of the Navier-Stokes equations and the Taylor polynomial LES model blow up in finite time. The solution obtained with the Smagorinsky model possesses a nearly constant total kinetic energy. One gets an almost steady state solution which is certainly not correct for $R e=10,000$. Only both types of the rational LES model compute unsteady solutions with bounded total kinetic energy which is qualitatively correct. However, the results for both variants of the rational LES model differ more than in the $2 \mathrm{D}$
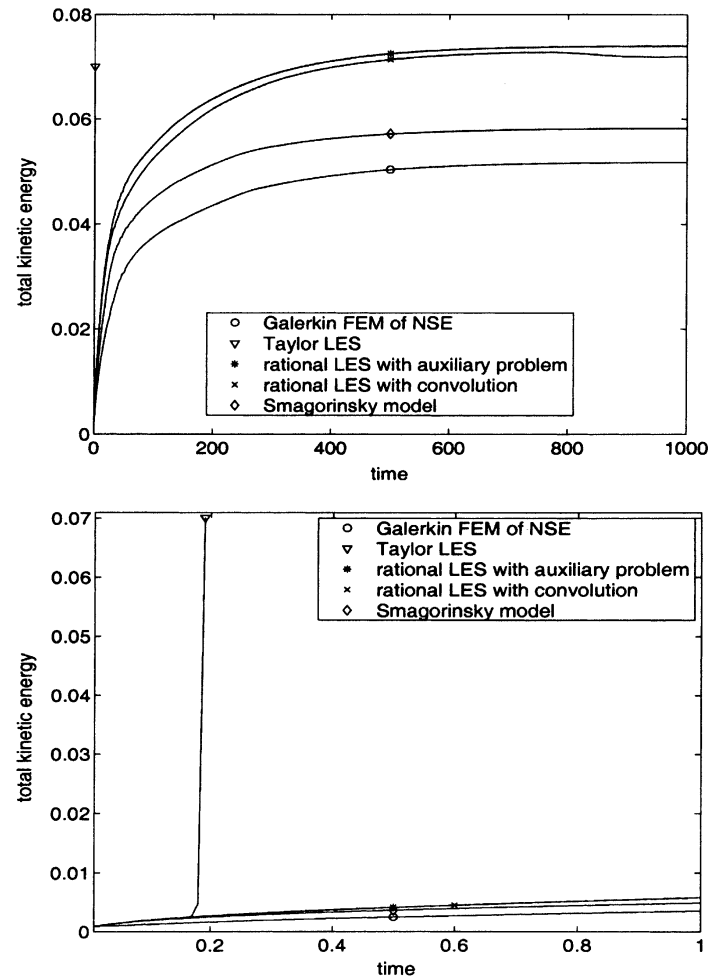

FIGURE 8 2D driven cavity problem, total kinetic energy, $R e=$ $10,000, h=1 / 64, \delta=\sqrt{2} / 64$. Note the blow up of the Taylor LES model.

computations. We think that the reason of these differences is related to the auxiliary problem. The explicit convolution and the auxiliary elliptic solution differ mostly near the boundary. The boundary has a greater influence in $3 \mathrm{D}$ on coarse meshes than in $2 \mathrm{D}$. Both variants of the rational LES model vary above all in such cells due to the artificial boundary condition of the auxiliary problem. Cutplanes of the flow fields obtained with the considered LES models are presented in Fig. 10.

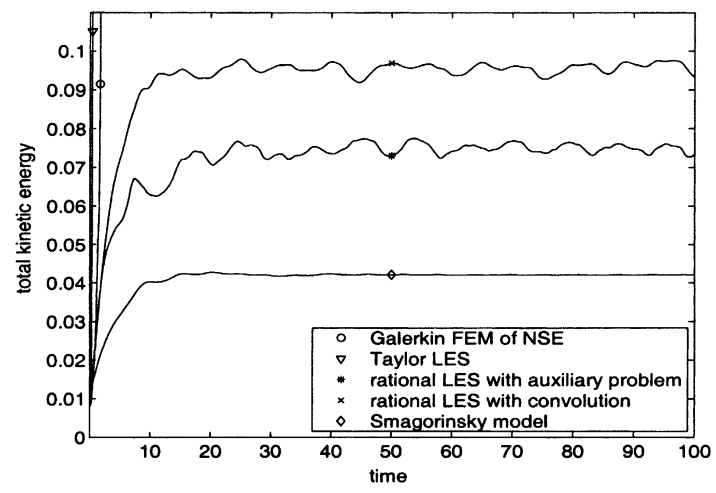

FIGURE 9 3D driven cavity problem, total kinetic energy, $R e=$ $10,000, h=1 / 8, \delta=\sqrt{3} / 8$. 

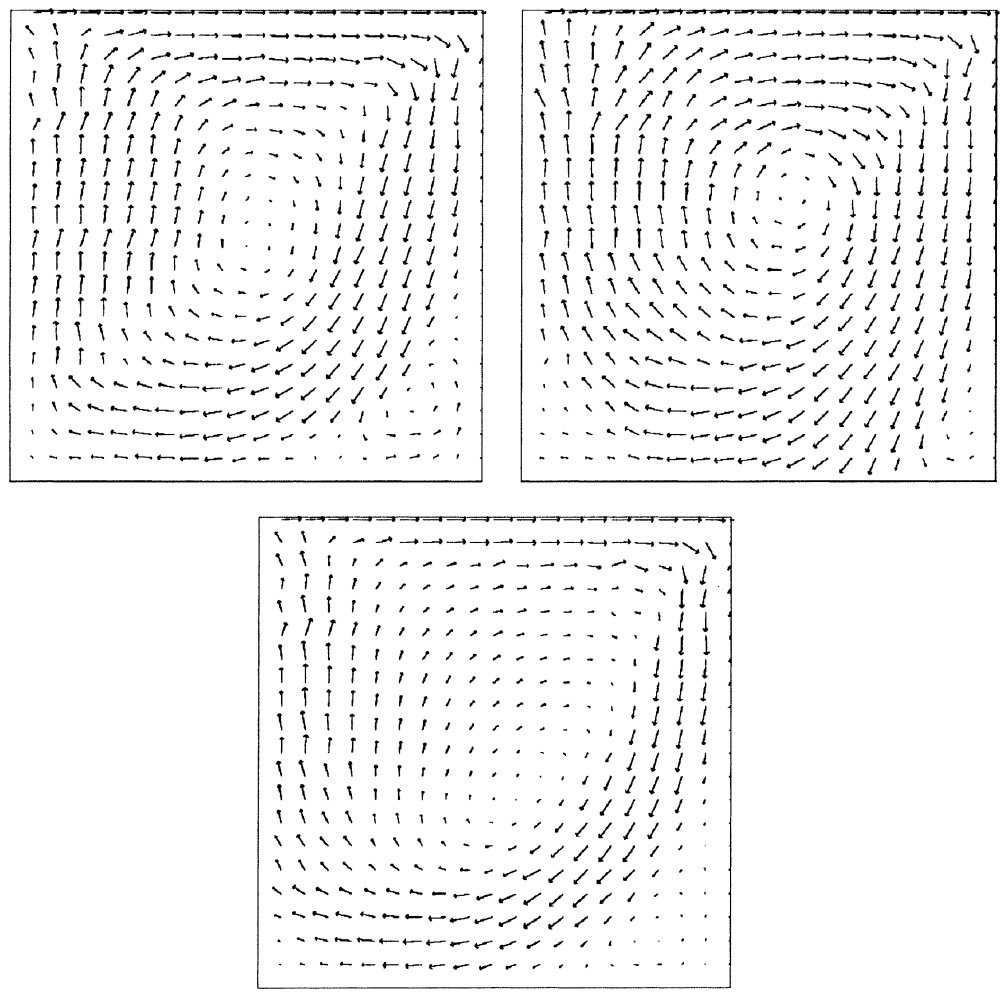

FIGURE 10 3D driven cavity problem, velocity field in the plane $y=0.5, R e=10,000, h=1 / 8, \delta=\sqrt{3} / 8$ and $t=100$ : upper left: rational LES model with auxiliary problem, upper right: rational LES model with convolution, lower: Smagorinsky model.

\section{CONCLUSIONS}

This section summarises the most important results of our study.

- Both types of the rational LES model with turbulent viscosity of Smagorinsky type behaved qualitatively correctly for high Reynolds number driven cavity flows. The main eddy of these flows is computed on a coarse grid much better compared to the Smagorinsky model.

- The rational LES model at high Reynolds number still requires a turbulent fluctuation model to prevent blow up. The magnitude of turbulent fluctuation required is much smaller than for the Taylor LES model.

- The Taylor LES model appeared to have an incorrect total kinetic energy balance leading to nearly immediate finite time blow up of its total kinetic energy in high Reynolds number flow computations. This blow up occurs even including a Smagorinsky model for $\overline{\mathbf{u}^{\prime} \mathbf{u}^{\prime}}$. The Taylor LES model is not suited to model turbulent flows.

- Laminar driven cavity flow was replicated well by the Taylor LES model and both types of the rational LES model.

This first numerical study shows that the rational LES model fulfils some necessary requirements which should be satisfied for a useful LES model. Further studies, including quantitative investigations of solutions, are needed to explore the full potential of this model. Such investigations are subject of a forthcoming study.

\section{Acknowledgements}

The first author was partially supported by NSF grants INT 9814115, and INT 9805563. The second, fourth and fifth authors were partially supported by the DAAD (Deutsche Akademische Austauschdienst). The fourth author was partially supported in addition by the Deutsche Forschungsgemeinschaft (DFG) by grant FOR 301/2-1. The third author was partially supported by NSF grants INT 9814115, DMS 9972622 and INT 9805563.

\section{References}

Aldama, A.A. (1990) Filtering Techniques for Turbulent Flow Simulation, Volume 56 of Springer Lecture Notes in Eng. (Springer, Berlin).

Bristeau, M.O., Glowinski, R. and Periaux, J. (1987) "Numerical methods for the Navier-Stokes equations: applications to the simulation of compressible and incompressible viscous flows", Comput. Phys. Rep. 6, 73-187.

Cantekin, M.E., Westerink, J.J. and Luettich, Jr., R.A. (1994) "Low and moderate Reynolds number transient flow simulations using space 
filtered Navier-Stokes equations", Numer. Methods Part. Diff. Equ. 10, 491-524.

Clark, R.A., Ferziger, J.H. and Reynolds, W.C. (1979) "Evaluation of subgrid-scale models using an accurately simulated turbulent flow", J. Fluid Mech. 91, 1-16.

Coletti, P. (1998) "Analytic and Numerical Results for $k-\varepsilon$ and Large Eddy Simulation Turbulence Models" PhD Thesis, University of Trento, Italy.

Fortin, M. (1993) "Finite element solution of the Navier-Stokes equations", In: Iserles, A., ed, Acta Numerica (Cambridge University Press), pp. 239-284.

Galdi, P. and Layton, W.J. (2000) "Approximation of the larger eddies in fluid motion II: a model for space filtered flow", Math. Models Methods Appl. Sci. 10(3), 343-350.

Germano, M. (1986) "A proposal for a redefinition of the turbulent stresses in the filtered Navier-Stokes equations", Phys. Fluids 29, 2323-2324.

Germano, M., Piomelli, U., Moin, P. and Cabot, W. (1991) "A dynamic subgridscale eddy viscosity model", Phys. Fluids A 3, 1760-1765.

Ghia, U., Ghia, K.N. and Shin, C.T. (1982) "High-Re solutions for incompressible flow using the Navier-Stokes equations and a multigrid method", J. Comput. Phys. 48, 387-411.

Gresho, P.M. and Sani, R.L. (1998) "Problems and solutions (generalized and fem) related to rapid and impulsive changes for incompressible flows", In: Hafez, M. and Oshima, K., eds, Computational Fluid Dynamics Review 1998 (World Scientific, Singapore) Vol. II, pp. 614-642.

Gresho, P.M. and Sani, R.L. (2000) Incompressible Flow and the Finite Element Method (Wiley, Chichester).

Iliescu, T. and Layton, W.J. (1998) "Approximating the larger eddies in fluid motion III: the Boussinesq model for turbulent fluctuations", Analele Stiințifice ale Universității Al. I. Cuza Iași, Tomul XLIV, s.I.a, Matematică 44, 245-261.

John, V. (2002) "Higher order finite element methods and multigrid solves in a benchmark problem for the 3D Navier-Stokes equations", Int. J. Numer. Methods Fluids, 40, 775-798.
John, V. and Matthies, G. (2002) "Higher order finite element discretizations in a benchmark problem for incompressible flows", Int. J. Numer. Methods Fluids 37, 885-903.

John, V. and Tobiska, L. (2002) "Smoothers in coupled multigrid methods for the parallel solution of the incompressible NavierStokes equations", Int. J. Numer. Methods Fluids 33, 453-473.

Klouček, P. and Rys, F.S. (1994) "Stability of the fractional step $\theta$-scheme for the nonstationary Navier-Stokes equations", SIAM Numer. Anal. 31, 1312-1335.

Ladyzhenskaya, O.A. (1967) "New equations for the description of motion of viscous incompressible fluids and solvability in the large of boundary value problems for them", Proc. Steklov Inst. Math. 102, $95-118$.

Leonard, A. (1974) "Energy cascade in large eddy simulation of turbulent fluid flows", Adv. Geophys. 18A, 237-248.

Matthies, G. and Tobiska, L. (2002) "The inf-sup condition for the mapped $Q_{k}-P_{k-1}^{\text {disc }}$ element in arbitrary space dimensions", Computing, In press.

Pope, S.B. (2000) Turbulent Flows (Cambridge University Press).

Rannacher, R. (2000) "Finite element methods for the incompressible Navier-Stokes equations", In: Galdi, G.P., Heywood, J.G. an Rannacher, R., eds, Fundamental Directions in Mathematical Fluid Dynamics (Birkhäuser), pp. 191-293.

Rodi, W., Ferziger, J.H., Breuer, M. and Pourquié, M. (1997) "Status of large eddy simulation: results of a workshop", J. Fluids Eng. 119 $248-262$.

Sagaut, P. (2001) Large Eddy Simulation for Incompressible Flows (Springer, Berlin/Heidelberg/New York).

Smagorinsky, J.S. (1963) "General circulation experiments with the primitive equations", Mon. Weather Rev. 91, 99-164.

Turek, S. (1999) Efficient Solvers for Incompressible Flow Problems: An Algorithmic and Computational Approach, Volume 6 of Lecture Notes in Computational Science and Engineering (Springer, Berlin).

Zang, Y., Street, R.L. and Koseff, J.R. (1993) "A dynamic mixed subgrid-scale model and its application to turbulent recirculating flows", Phys. Fluids A 5, 3186-3196. 